\title{
Ripples in spacetime
}

\author{
The 2017 Nobel prize in Physics has been awarded to Rainer Weiss, Barry C. Barish and Kip S. Thorne \\ "for decisive contributions to the LIGO detector and the observation of gravitational waves".
}

It is, frankly, difficult to find something original to say about the detection of gravitational waves that hasn't been said already. The technological feat of measuring fluctuations in the fabric of spacetime less than one-thousandth the width of an atomic nucleus is quite simply astonishing. The scientific achievement represented by the confirmation of a century-old prediction by Albert Einstein is unique. And the collaborative effort that made the discovery possible - the Laser Interferometer Gravitational-Wave Observatory (LIGO) is inspiring.

Rainer Weiss and Kip Thorne were, along with the late Ronald Drever, founders of the project that eventually became known as LIGO. In the 1960s, Thorne, a black hole expert, had come to believe that his objects of interest should be detectable as gravitational waves. Separately, and inspired by previous proposals, Weiss came up with the first calculations detailing how an interferometer could be used to detect them in 1972. By the early 1980s they had joined forces with Drever to work on the giant twin detectors that would eventually be set up in Livingston, Louisiana, and Hanford, Washington. This not only required scientific and technological vision, but also political skill and pragmatism to get the project funded by the US National Science Foundation (and eventually by an international consortium of funding agencies).

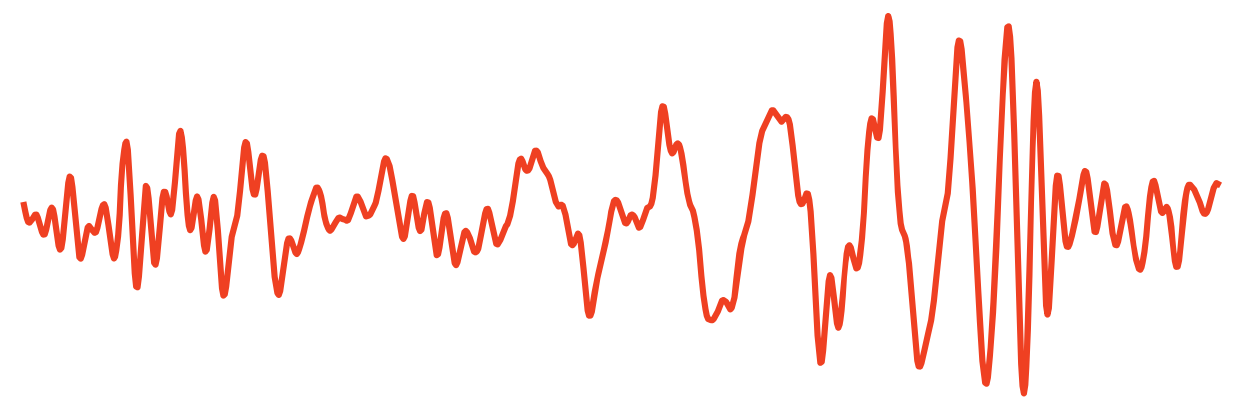

Adapted from Phys. Rev. Lett. 116, 061102 (2016), under Creative Commons Licence.

Barry Barish, who was the director of LIGO from 1997 to 2005, is widely credited with transforming it into a 'big physics' collaboration, and providing the organizational structure required to ensure it worked. Of course, the passion, skill and dedication of the thousand or so scientists that worked on LIGO over many decades was essential to its scientific success - an aspect all three laureates and the Nobel committee itself have been keen to stress.

The discovery of GW150914 (pictured), the echo from a collision of two black holes about 1.3 billion light years away, caused a media sensation when it was announced in February last year. It was, rightly, heralded as the dawn of a revolution in astrophysics.
Last month we received a spectacular demonstration that talk of a new era of gravitational astronomy was no exaggeration. Cued by detections at LIGO and Virgo, an interferometer based in Pisa, Italy, more than 70 teams of researchers working at different telescopes around the world confirmed the observation of a neutron-star merger. A slew of papers published on 16 October reported the electromagnetic counterpart to the gravitational-wave detection.

These observations simultaneously solve a number of outstanding problems, including the origin of the heavier elements of the periodic table. It is difficult to imagine that this is the last time that ripples of spacetime will reach Stockholm.

\section{A matter of quantum}

\section{Presenting an Insight on quantum materials.}

At Nature Physics we enjoy putting together the occasional Insight consisting of a number of authoritative Reviews covering an important topic in physics. This month, we've joined forces with Nature Materials to produce an Insight on quantum materials (http://go.nature.com/2hNXJHa) - an emerging theme that we've already picked up on in the recent past (Nat. Phys. 12, $105 ; 2016)$.

We are particularly pleased to acknowledge our sponsors, the Gordon and Betty Moore Foundation and the Simons Foundation. Thanks to their support, we are able to offer free online access of the Insight for twelve months, in addition to distributing print copies at the upcoming Materials Research Society fall meeting in Boston and the American Physical Society March meeting in Los Angeles.

Finding a definition for quantum materials that is unanimously agreed on is tricky, but it seems like a growing number of scientists working at the frontiers of physics and materials science know a quantum material when they see one. This Insight attempts to define the field in more detail by examining the physics of quantum materials, their synthesis and design, the control over their properties, and the functionality they give rise to.

Of course we cannot hope to provide a fully comprehensive overview of the entire field in a single supplement, but we do hope it will prove an inspiring reflection of the field. Enjoy. 